\title{
The first high-quality chromosomal genome assembly of a medicinal and edible plant Arctium lappa
}

\author{
Liang $\mathrm{Xu}^{1}$, Shengnan $\mathrm{Li}^{2}$, Yanyun Yang${ }^{1}$, Yanping Xing ${ }^{1}$, Zhongren Zhang ${ }^{3}$, Tao Liu ${ }^{1}$, \\ Wuliji Ao ${ }^{1}$, Guihua Bao ${ }^{1}$, Zhilai Zhan ${ }^{1}$, Rong Zhao ${ }^{1}$, Tingting Zhang ${ }^{1}$, Dachuang Zhang ${ }^{1}$, \\ Tingguo Kang ${ }^{1}$, Yueyue Song ${ }^{1}$, and Che Bian ${ }^{1}$ \\ ${ }^{1}$ Affiliation not available \\ ${ }^{2}$ Liaoning University of Traditional Chinese Medicine - Dalian Campus \\ ${ }^{3}$ Novogene Bioinformatics Institute
}

May 4, 2021

\begin{abstract}
Arctium lappa has a long medicinal and edible history with great economic importance. We combined Illumina and PacBio sequences to generate the first high-quality chromosome-level draft genome of A. lappa. The assembled genome is approximately $1.79 \mathrm{~Gb}$ with a N50 contig size of $6.88 \mathrm{Mb}$. Approximately $1.70 \mathrm{~Gb}(95.4 \%)$ of the contig sequences were anchored onto 18 chromosomes using Hi-C data; the scaffold N50 was improved to be $91.64 \mathrm{Mb}$. Furthermore, we obtained 1.12 Gb (68.46\%) of repetitive sequences and 32,771 protein-coding genes; 616 positively selected candidate genes were identified. Additionally, we compared the transcriptomes of A. lappa roots at three different developmental stages and identified 8,943 differentially expressed genes (DEGs) in these tissues. Among candidate genes related to lignan biosynthesis, the following were found to be highly correlated with the accumulation of arctiin: 4-coumarate-CoA ligase (4CL), dirigent protein (DIR), and hydroxycinnamoyl transferase (HCT). These data can be utilized to identify genes related to A. lappa quality or provide a basis for molecular identification and comparative genomics among related species.
\end{abstract}

\section{Hosted file}

manuscript.pdf available at https://authorea.com/users/411779/articles/520689-the-firsthigh-quality-chromosomal-genome-assembly-of-a-medicinal-and-edible-plant-arctium-lappa 